УДК 330.35

$10.17213 / 2075-2067-2021-1-167-177$

\title{
ПРОЕКТИРОВАНИЕ ПРОИЗВОДСТВЕННЫХ СИСТЕМ, ОБЕСПЕЧИВАЮЩИХ СОЦИАЛЬНЫЕ И ЭКОНОМИЧЕСКИЕ ИНТЕРЕСЫ ОБЩЕСТВА И ГОСУДАРСТВА ${ }^{1}$
}

\author{
(C) 2021 г. A. А. Пахомова , В. Е. Федорчук*, И. Кадар \\ "Южсно-Российский государственный политехнический университет (НПИ) \\ имени М. И. Платова, г. Новочеркасск, Россия \\ ** Будапештский университет технологий и экономики, г. Будапешт, Венгрия
}

Целью исследования является разработка методологии проектирования производственных и технических систем, обеспечивающей социальные и экономические интересы общества и государства, проявляющиеся в наращивании человеческого и сочиильного капитала и переходе российской промышленности к новым передовым интеллектуальным и циирровым технологиям.

Методологическую базу исследования представляют теории инновачионного развития, производственных систем, NBIC-конвергенции, теории развития Индустрии 4.0, которые рассматривают различные инновачии как основныле факторы экономического роста, с учетом повышения уровня рисков и угроз различного характера. Исследования данных теорий позволяют предложить методы проектирования отдельных элементов производственных систем.

Результаты исследования. Использование в методологии проектирования производственных и технических систем разработанной человекоориентированной концепции управления HR-ресурсами в условиях NBIC-конвергенции имеет все инструменты для итерационного саморазвития и самосовершенствования, обеспечивая безопасные условия труда и постоянный контроль здоровья человека, что позволяет максимизировать процент отдачи от человеческого ресурса и снизить уровень оппортунистического поведения.

Данный инструмент является замкнутой системой, а следовательно, имеет определённый предел в своём развитии. Для перехода на новый этап человеку нужно выйти из системы для формирования новых смыслов и конщепций, что невозможно без гуманитарной и культурной составляющей системной парадигмы, а также без взаимодействия между научно-производственными кластерами.

Человечество приближсается к моменту перехода от механистической парадигмы кхолистической, от энтропийной стадии к синтропической. В настоящзее время циивилизация находится на энтропийной стадии, поскольку ориентирована в первую очередь на прибыль. Трансформация связана с человеческим разумом, а разум в первую очередь связан с синтропией, созданием новой парадигмы и преодолением энтропии, что подтверждает необходимость формирования правильной системь иенностей. Для достижения уникального спиралевидного роста необходимо разорвать сопротивление среднего значения текущего баланса познания и дойти до критической (начальной) точки спирального роста.

Таким образом, предлагается модель развития по спирали, каждый виток которой будет представлять собой самоорганизуюшуюся систему, обеспечиваюшую социальные

1 Исследование выполнено при финансовой поддержке РФФИ и РЯИК в рамках научного проекта 20-51023002 «Развитие системной парадигмы Яноша Корнаи - фундаментальная основа создания производственных и технических систем в условиях NBIC-конвергенции». 
и экономические интересы общества и государства, проявляющиеся в наращивании человеческого и сочиального капитала и переходе российской промышленности к новым передовым интеллектуальным и ичифровым технологиям.

Перспективу исследования составляет разработка управленческого инструментария, обеспечивающего решение задач проектирования в условиях NBIC-конвергенции на основе соииальных иелей, их трансформирования в экономические характеристики, на основе которых формируются организачионно-технические решения.

Ключевые слова: NBIC-конвергенция; проектирование; технические системы; инновации; экономический рост; социально-экономическое развитие.

\title{
DESIGN OF PRODUCTION SYSTEMS THAT ENSURE THE SOCIAL AND ECONOMIC INTERESTS OF SOCIETY AND THE STATE
}

\author{
(C) 2021 A. A. Pakhomova*, V. E. Fedorchuk*, I. Kadar** \\ "Platov South Russian State Polytechnic University (NPI), Novocherkassk, Russia \\ ${ }^{* *}$ Budapest University of Technology and Economics, Budapest, Hungary
}

The aim of the study is to develop a methodology for designing production and technical systems that ensures the social and economic interests of society and the state, which are manifested in the increase of human and social capital and the transition of Russian industry to new advanced intellectual and digital technologies.

The methodological basis of the research is represented by the theories of innovative development, production systems, NBIC-convergence, and the theory of Industry 4.0 development, which consider various innovations as the main factors of economic growth, taking into account the increase in the level of risks and threats of various nature. Studies of these theories allow us to propose methods for designing individual elements of production systems.

Results of the study. The use of the developed human-oriented concept of HR-resource management in the context of NBIC-convergence in the design methodology of production and technical systems has all the tools for iterative self-development and self-improvement, providing safe working conditions and constant monitoring of human health, which allows you to maximize the percentage of return on human resources and reduce the level of opportunistic behavior.

This tool as a system is closed, and therefore has a certain limit in its development. To move to a new stage, a person needs to leave the system to form new meanings and concepts, which is impossible without the humanitarian and cultural component of the system paradigm, as well as without interaction between scientific and industrial clusters.

Humanity is approaching the moment of transition from the mechanistic to the holistic paradigm, from the entropic to the syntropic stage. Currently, civilization is at the entropy stage, since it is primarily focused on profit. Transformation is associated with the human mind, and the mind is primarily associated with syntropy, the creation of a new paradigm and overcoming entropy, which confirms the need for the formation of a correct value system. To achieve a unique spiral growth, it is necessary to break the resistance of the average value of the current balance of knowledge and reach the critical (initial) point of spiral growth.

Thus, we propose a model of development in a spiral, each turn of which will be a self-organizing system that ensures the social and economic interests of society and the state, manifested in the increase of human and social capital and the transition of Russian industry to new advanced intellectual and digital technologies. 
Future research is development of managerial tools ensuring the solution of design tasks in terms of NBIC-convergence on the basis of social objectives, their transformation into economic characteristics, upon which to build organizational and technical solutions.

Key words: NBIC-convergence; design; technical systems; innovation; economic growth; socio-economic development.

Введение. Отставание российской экономики в технологической сфере обуславливает низкую конкурентоспособность российской национальной экономики и представляет в настоящее время реальную угрозу безопасности суверенитету страны и перспективам ее развития в будущем. Это требует разработки эффективных методов проектирования новых производственных систем практически во всех отраслях российского народного хозяйства в соответствии с современными тенденциями, связанными с процессами реиндустриализации, цифровизации производства, формирования экономики знаний, при этом ключевым сегментом могут стать NBIC-технологии.

Изменения, вносимые в современное общество технологическим прогрессом, способны уже в обозримой перспективе привести к серьезным изменениям экономических основ общества в целом и производственных систем в частности. Особенно интересным и значимым представляется взаимовлияние информационных технологий, биотехнологий, нанотехнологий и когнитивных наук. Данное явление, не так давно замеченное исследователями, получило название NBICконвергенции.

Одним из вариантов решения таких задач является использование NBIC-технологии. Считаем необходимым напомнить, что свое название NBIC-конвенгерция получила по первым буквам основополагающих изменений технологий (N - нано, В - био, $\mathrm{I}$ - инфо, C — когно). Конвергенция означает не только взаимное влияние, но и взаимопроникновение технологий, когда границы между отдельными технологиями стираются, а многие интересные результаты возникают именно в рамках междисциплинарной работы на стыке областей $[5,13]$. В настоящее время необходимо сокращение сроков разработки и осуществления таких проектов, оперативность в принятии и реализации технических и управленческих решений, преодоление инертности, традиционно присущей

Таблица 1

Типизация глобальных угроз, вызванных развитием NBIC-технологии

\begin{tabular}{|l|l|}
\hline \multicolumn{1}{|c|}{ Вид угрозы } & \multicolumn{1}{|c|}{ Характер и последствия реализации угроз } \\
\hline $\begin{array}{l}\text { Многокомпонентность } \\
\text { технологий }\end{array}$ & $\begin{array}{l}\text { Множественный характер технологий, размытые границы между } \\
\text { гражданским и военным применением и, как следствие, неэффек- } \\
\text { тивность существующих средств и технологий контроля }\end{array}$ \\
\hline $\begin{array}{l}\text { Прорывные результаты } \\
\text { науки }\end{array}$ & $\begin{array}{l}\text { Невозможность предугадать все последствия выхода искусствен- } \\
\text { ных живых систем в окружающую среду }\end{array}$ \\
\hline $\begin{array}{l}\text { Многовариантность } \\
\text { использования }\end{array}$ & $\begin{array}{l}\text { Биогенетические нанобиотехнологии, позволяющие продуциро- } \\
\text { вать искусственные живые системы с заданными свойствами, на } \\
\text { основе чего могут быть созданы как сверхэффективные лекарс- } \\
\text { тва, так и средства поражения }\end{array}$ \\
\hline $\begin{array}{l}\text { Психофизиологическая } \\
\text { трансформация }\end{array}$ & $\begin{array}{l}\text { Когнитивные технологии, основанные на конвергенции инфоког- } \\
\text { нитивных и социогуманитарных наук и воздействующие на пси- } \\
\text { хофизиологическую сферу человека с целью контроля и управле- } \\
\text { ния его сознанием и телом }\end{array}$ \\
\hline
\end{tabular}


субъектам бизнеса. Кроме того, следует отметить, что высокотехнологические производственные системы являются «точками роста» человеческого капитала, привлекающими многих перспективных исследователей и разработчиков, рассчитывающих на скорейшую коммерциализацию своих идей и проектов.

В то же время нельзя не отметить, что нарастающие риски и угрозы, глобальные вызовы различного характера не могут найти решения по прежним фундаментальным лекалам парадигмы развития нашей цивилизации. В результате ученые в различных направлениях науки приходят к выводу о необходимости поиска новых способов и методов решения поставленных задач функционирования производственных систем в новых реалиях, сталкиваясь с новыми видами рисков и угроз (таблица 1).

Технологическая эволюция способствует увеличению структурной и динамической сложности, вызванной неоднородностью и непредвиденным поведением компонентов системы, требующих существенных изменений в сложившейся парадигме. В этой связи усиливается роль научно-исследовательских работ в данном направлении.

\section{0}

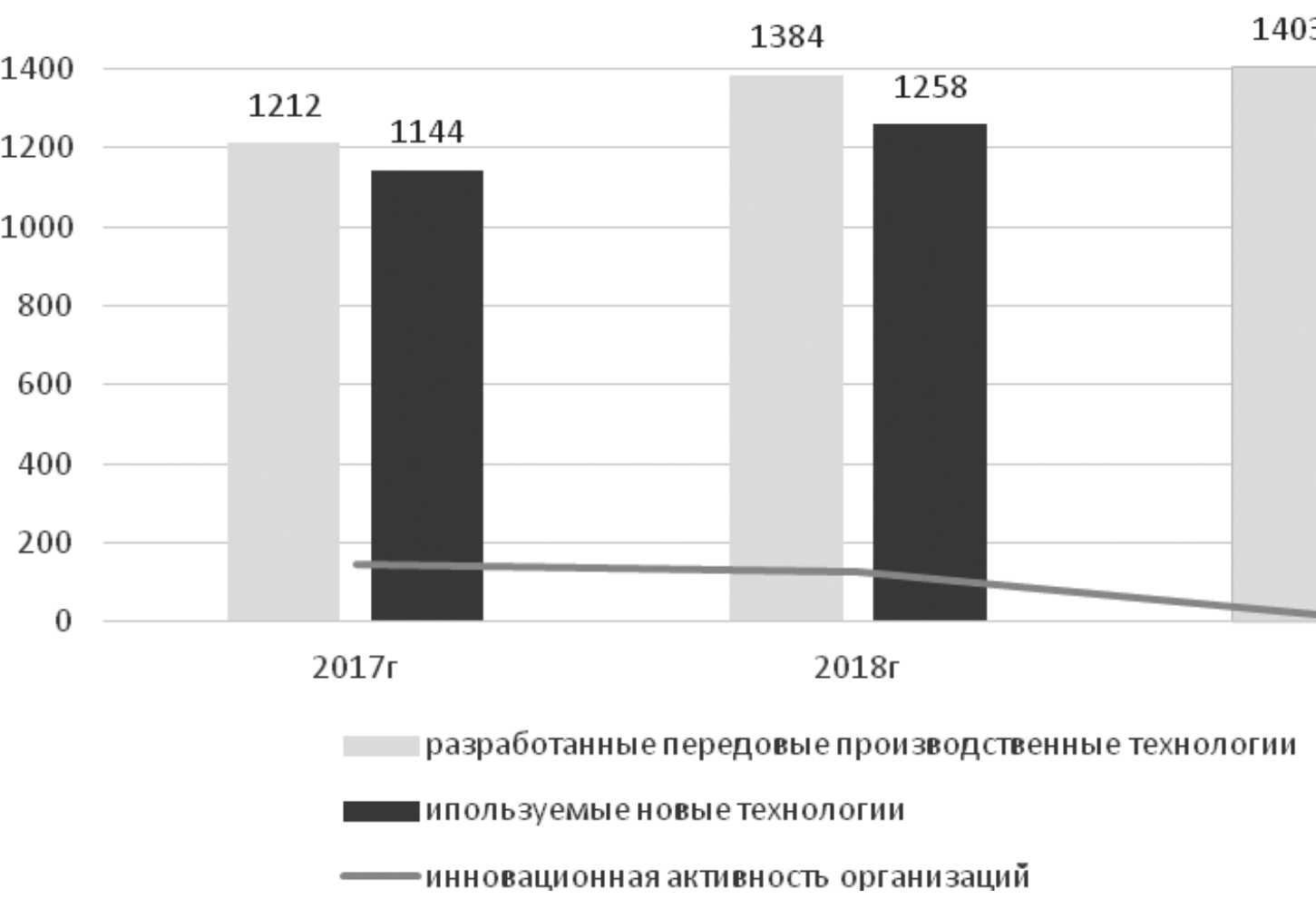

Рис. 1. Динамика показателей технологического развития Российской Федерации (по данным Росстат РФ, https://rosstat.gov.ru)
Материалы и методы исследования. Проведенные исследования международной литературы показывают увеличение количества научно-исследовательских работ в сфере NBIC-технологий в мире [19]. В рамках нашего исследования были проанализированы статистические данные, представленные Федеральной службой государственной статистики Российской федерации по количеству разрабатываемых передовых производственных технологий, используемых передовых производственных технологий и инновационной активности (рисунок 1).

Статистические данные показывают сокращение до 4\% ежегодно используемых технологий и уровня инновационной активности, количества организаций выполняющих НИОКР при росте разрабатываемых передовых производственных технологий, что, несомненно, с одной стороны свидетельствует о потенциале, с другой - о кризисных явлениях в производственных системах.

Динамика финансирования научных исследований в Российской Федерации представлена на рисунке 2.

Финансирование научных исследований из средств федерального бюджета послед-

\section{4} 1228

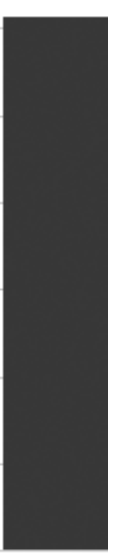

2019 r 


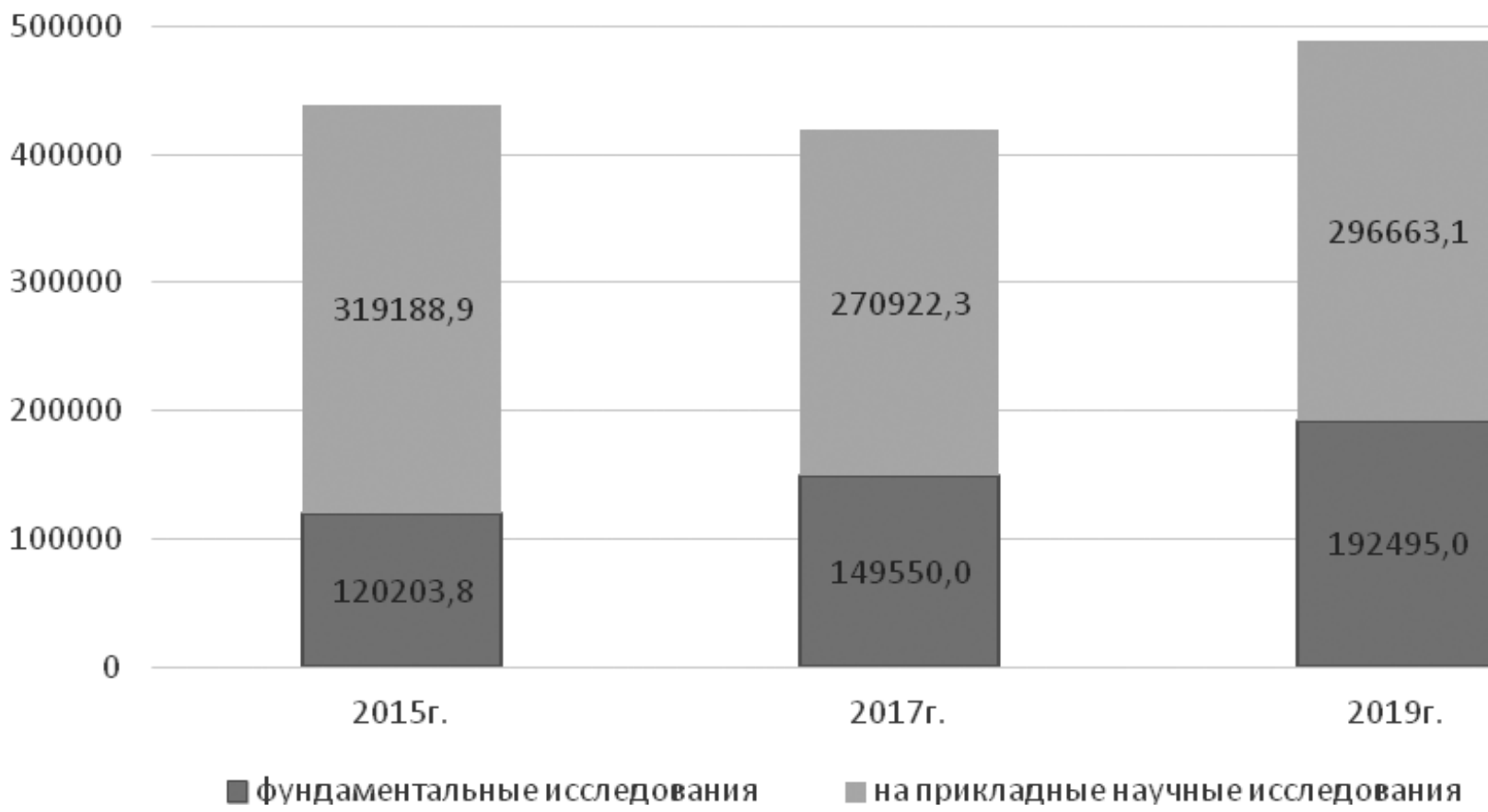

Рис. 2. Динамика финансирования научных исследований в Российской Федерации (млн. руб.) (по данным Росстат РФ, https://rosstat.gov.ru)

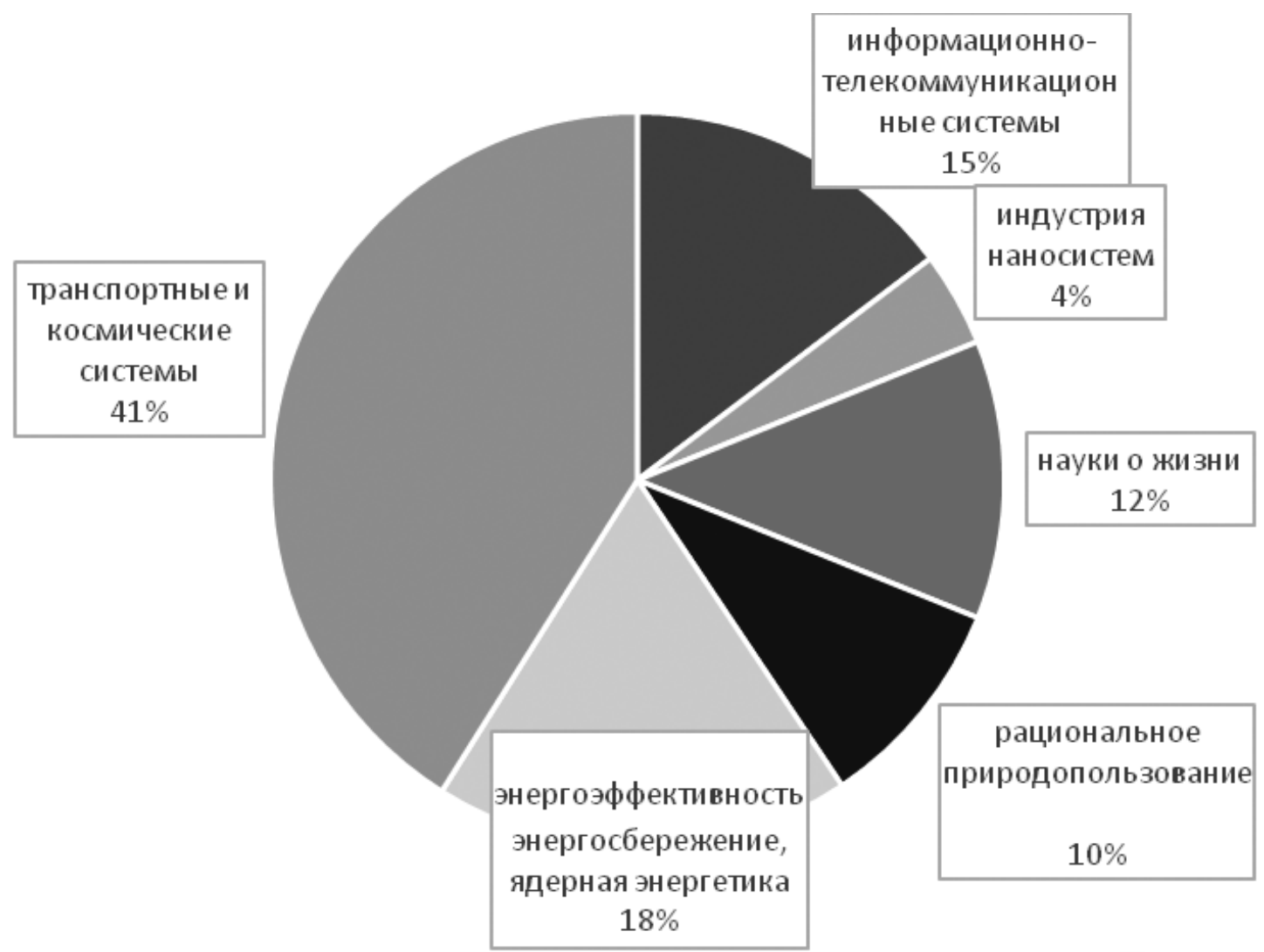

Рис. 3. Затраты на научные исследования и разработки по приоритетным направлениям развития науки, технологий и техники в Российской Федерации в 2019 г. (по данным Росстат РФ, https://rosstat.gov.ru) 
ние 10 лет имеет стабильный рост. Так, если в 2010 году выделено 17396,4 млн. руб, что составило 1,69\% расходов федерального бюджета, то в 2019 году финансирование выросло более чем в 28 раз и составило 489158,4 млн. руб (2,69\%). При этом большая доля отводится на финансирование прикладных научных исследований.

Структура затрат на научные исследования и разработки по приоритетным направлениям развития науки, технологий и техники в Российской Федерации представлена на рисунке 3.

В 2019 году, как и на протяжении последних пяти лет, наибольший удельный вес в структуре затрат отводится на научные исследования и разработки в сфере транспортных и космических систем - 247266,6 млн. руб., энергоэффективность, энергосбережение, ядерную энергетику — 110366,7 млн. руб.

Структура организаций, выполняющих научно-исследовательские работы, свидетельствует об институциональных изменениях, произошедших в последнее десятилетие (таблица 2).

Количество некоммерческих организаций, выполняющих НИКР за последние двадцать лет, увеличилось более чем в 3 раза, на втором месте стоит динамика высшего образования.

Как показывают глобальные тенденции развития инновационной сферы в промышленно развитых странах, важнейшим элементом политики в последние годы становится совершенствование и изменение всей системы институтов, связанных с инновационной сферой. Идет активная институциональная реформа в области организационно-управленческих подсистем национальных инно- вационных систем, нормативно-правовой инновационной инфраструктуры, формируются наиболее благоприятные условия для науки и бизнеса, нормативно-правовой базы партнерства государства и частного сектора, что наиболее характерно для инновационной сферы деятельности. В России в последнее десятилетие также происходят институциональные изменения, направленные на развитие и повышение конкурентоспособности современных высокотехнологичных и наукоемких производств. Однако, единого системного подхода в данный момент не создано. Для решения поставленной задачи следует разработать методологию, которая позволила бы проектировать эффективные и безопасные производственные системы в условиях NBIC-конвергенции, обеспечивающей социальные и экономические интересы общества и государства, проявляющиеся в наращивании человеческого и социального капитала и переходе российской промышленности к новым передовым интеллектуальным и цифровым технологиям, основной которой может стать парадигма Яноша Корнаи.

Развитие системной парадигмы Яноша Корнаи - фундаментальная основа создания производственных и технических систем в условиях NBIC-конвергенции. В основе предлагаемой методологии лежит системная парадигма Я. Корнаи [19], развитая в последующих исследованиях [13], - концепция видения объекта и предмета исследований, согласно которой социально-экономическое пространство рассматривается как единая система, заключающая в себе множество относительно самостоя-

Количество организаций, выполнявших научные исследования и разработки, по секторам деятельности по Российской Федерации

\begin{tabular}{|l|c|c|c|c|c|c|c|}
\hline \multicolumn{1}{|c|}{ Показатель } & 2000 г. & 2010 г. & 2015 г. & 2016 г. & 2017 г. & 2018 г. & 2019 г. \\
\hline $\begin{array}{l}\text { Число организаций — всего, в } \\
\text { т.ч. по секторам деятельности: }\end{array}$ & 4099 & 3492 & 4175 & 4032 & 3944 & 3950 & 4051 \\
\hline государственный & 1247 & 1400 & 1560 & 1546 & 1493 & 1511 & 1479 \\
\hline предпринимательский & 2278 & 1405 & 1400 & 1326 & 1292 & 1304 & 1374 \\
\hline высшего образования & 526 & 617 & 1124 & 1064 & 1038 & 998 & 1057 \\
\hline некоммерческих организаций & 48 & 70 & 91 & 96 & 121 & 137 & 141 \\
\hline
\end{tabular}


тельных подсистем, состав и структура которых определяется в соответствии с позицией наблюдателя или группы наблюдателей. Причины возникновения и распространения системной парадигмы были связаны с кризисом ортодоксальной экономической теории. При этом под социально-экономической системой понимается относительно обособленная и устойчивая часть или определенный аспект социально-экономического пространственно-временного континуума, для которой характерны внешняя целостность и внутреннее многообразие. В рамках этой парадигмы любая техническая система (которая по сути является социотехнической, ибо предусматривает участие человека в ее создании и использовании) может рассматриваться как именно такая подсистема, находящаяся в некотором социально-экономическом пространстве. Системная парадигма Я. Корнаи была положена в основу понимания производственной системы как мультипространственной системы, объединяющей в себе разнородные элементы и ресурсы [17].

Мультипространственные производственные системы являются особым видом систем, которые могут быть описаны с помощью метрик, форм, координат, системой взаимодействия и т.п. Мультипространственые системы описаны достаточно широко, но являются одними из малоисследованных, требующих формирования целевого методологического аппарата управления.

В рамках нашего исследования мы предлагаем проектирование производственных систем, обеспечивающих социальные и экономические интересы общества и государства представить как мультипространственную задачу с неопределенным количеством неизвестных, однако имеющих заданное конечное целеполагание.

Ключевым элементом NBIC-конвенгерции, по нашему мнению, должен стать человек. Необходимо найти не только инструменты, позволяющие контролировать, оптимизировать и развивать производственную деятельность человека, но и механизмы формирования и эволюции культурно-мировозренческих взглядов и, как следствие, новых смыслов и концепций, позволяющих обеспечивать устойчивое развитие человечества и парадигмы науки. Для этого необходимо создание человекоориентированной концепции управления HR-ресурсами в условиях NBIC-конвергенции.

Человекоориентированная концепция управления HR-ресурсами в условиях NBIC-конвергенции. В рамках научного проекта 20-510-23002 «Развитие системной парадигмы Яноша Корнаи - фундаментальная основа создания производственных и технических систем в условия NBIC-конвергенции» кандидатом технических наук, доцентом кафедры «Нефтегазовые техника и технологии» ФГБОУ ВО «Южно-Российский государственный политехнический университет (НПИ) имени М.И. Платова» Е. В. Сидоровой была разработана человекоориентированная концепция управления HR-pecypcaми мультипространственной производственной системы в условиях NBIC-конвергенции. В разработанной человекоориентированной концепции управления HR-ресурсами мультипространственной производственной системы в условиях NBIC-конвергенции основными элементами являются «Человек» и «Машина». Их взаимодействие обусловлено двойными и глобальными итерационными связями. Каждому человеку соответствует машина (или их совокупность) в сложной системе координат и взаимосвязей. Человек осуществляет управление и обучение машины, реагируя обратной связью, передавая целевую информацию, принимая ответственность действия за результат и конечное целеполагание. Машина, в свою очередь, аккумулирует историю всех операций человека, параллельно взаимодействуя с другими машинами и анализируя их эффективность, исходя из заданной целевой производственной функции. Формируя базу знаний, а также на основе существующих моделей в мультипростанственной системе машина прогнозирует и предлагает человеку различные рациональные сценарии поведения. Таким образом, снижается риск оппортунистического поведения человека: все его действия подкреплены мультипространственным системным анализом. Взаимодействие «Человек»«Человек» необходимо для усовершенствования производственных алгоритмов, внесения в них большей доли когнитивной составляющей и корректировки действий в командной 
работе. Представленная модель позволяет спрогнозировать результат принятия тех или иных производственных решений либо предложить рациональные варианты сценариев, обеспечивающие оптимальные значения для заданных условий.

Заключение. Проблема проектирования мультипространственных производственных систем, обеспечивающих социальные и экономические интересы общества и государства, проявляющиеся в наращивании человеческого и социального капитала и переходе российской промышленности к новым передовым интеллектуальным и цифровым технологиям, имеет решение посредством релизации предлагаемой человекоориентированной концепции управления HR-ресурсами, развитие которой происходит по спирали. Каждый виток спирали представляет собой самоорганизующуюся систему. Для текущего этапа развития предложена киберфизическая система взаимодействия «Человек»-«Машина», базирующаяся на глобальных и двойных итерационных связях, параллельном анализе, прогнозировании сценариев, машинном обучении и когнитивной составляющей.

Данная концепция позволяет: контролировать, оптимизировать и развивать производственную деятельность человека, обеспечивать итерационное саморазвитие и самосовершенствование системы, гарантировать стабильность и безопасность работы системы, максимизировать процент отдачи от человеческого ресурса, снижать уровень оппортунистического поведения человека.

Переход на новый виток развития обеспечивается выходом из системы для формирования новых смыслов и концепций за счет гуманитарной и культурной составляющей, а также посредством взаимодействия между научно-производственными кластерами.

\section{Литература}

1. Angelopoulou A., Mykoniatis K., Boyapati N. R. Industry 4.0: The use of simulation for human reliability assessment // Procedia Manufacturing. - 2020. — №42. - P. 296-301. DOI:10.1016/j.promfg.2020.02.094.

2. Brocal F., González C., Komljenovic D., Katina P.F., Sebastián M.A. Emerg- ing Risk Management in Industry 4.0: An Approach to Improve Organizational and Human Performance in the Complex Systems // Complexity. — 2019. — №1-13. DOI:10.1155/2019/2089763.

3. Kolbachev E., Kolbacheva T. Human Factor and Working Out of NBIC-Technologies // AHFE 2018: Advances in Manufacturing, Production Management and Process Control. - 2019. - Vol. 793. - P. 179-190. DOI:10.1007/978-3-319-94196-7_17.

4. Maksimović M., Vujović V., Perišić B. Do It Yourself solution of Internet of Things Healthcare System: Measuring body parameters and environmental parameters affecting health // Journal of Information Systems Engineering \& Management. - 2016. - №1 (1). - P. 25-39. DOI:10.20897/lectito.201607.

5. Mikulášková J., Čambál M., Polakovič L', Urbanovičová $P$. Spiral Management: New Concept of the Social Systems Management// Networked Business Models in the Circular Economy. - 2020. - P. 174-199. DOI:10.2507/29th.daaam.proceedings.131.

6. Nocker M., Sena V. Big Data and Human Resources Management: The Rise of Talent Analytics // Social Sciences. — 2019. — №8(10). P. 273. - DOI:10.3390/socsci8100273.

7. Reunanen T. Human Factor in Time Management // Procedia Manufacturing. — 2015. №3. - P. 709-716. — DOI:10.1016/j.promfg.2015.07.311.

8. Robert M., Giuliani Ph., Gurau C. Implementing industry 4.0 real-time performance management systems: the case of Schneider Electric// Production Planning \& Control. - 2020. - DOI: 10.1080/09537287.2020.1810761.

9. Royakkers L., Timmer J., Kool L., Van Est R. Societal and ethical issues of digitization // Ethics and Information Technology. 2018. — №20. - P. 127-142. - DOI:10.1007/ s10676-018-9452-x.

10. Sassi I., Anter S., BekkhouchaA. An Overview of Big Data and Machine Learning Paradigms // Advances in Intelligent Systems and Computing. - 2019. — №915. - P. 237-251. DOI:10.1007/978-3-030-11928-7 21.

11. Som C., Hilty L.M. Qualitative risk assessment for converging technologies: nano-bioinfo-cogno technologies // Proceedings of International Symposium on EcoTopia Science. 2007. - P. 1178-1181. 
12. Tapus N., Manolache M.A. Integrated Decision Making using the Blockchain // Procedia Computer Science. - 2019. — №162. P.587-595. — DOI:10.1016/j.procs.2019.12.027.

13. Клейнер Г. Системная парадигма и теория предприятия // Вопросы экономики. 2002. - №10. - C. 24-33.

14. Ковальчук М. В., Нарайкин О.С. Природоподобные технологии - новые возможности и новые угрозы // Индекс безопасности. — 2016. — №22 (3-4). — С. 118-119.

15. Ковальчук M.B. Конвергенция наук и технологий - прорыв в будущее // Российские нанотехнологии. - 2011. №6(13-23). - C. 455-465. - DOI:10.31857/ S0869-5873895455-465.

16. Ковальчук М. В., Нарайкин О.С., Яичишина Е.Б. Природоподобные технологии: новые возможности и новые вызовы // Вестник Российской академии наук. - 2019. №89 (5). - C. 455-465. - DOI:10.31857/ S0869-5873895455-465.

17. Колбачев Е.Б. Производственные системы машиностроительных предприятий и их организационно-экономическая эволюция // Проблемы машиностроения и автоматизации. - 2016. - №2. - С. 12-16.

18. Корнаи Я. Системная парадигма // Вопросы экономики. - 2002. - №4. C. $18-26$.

19. Руденский О. В., Рыбак О. П. Инновационная цивилизация XXI века: конвергенция и синергия NBIC-технологий. Тенденции и прогнозы 2015-2030 [Электронный ресурс]// Информационно-аналитический бюллетень №3. - Режим доступа: http:// www.vixri.ru/wp-content/uploads/2011/08/ inf3_2010.pdf.

20. Медведев Д.А., Прайд Д. Феномен NBIC-конвергенции: Реальность и ожидания // Философские науки. — 2008. — №1 . C. 97-117.

21. Павельева Т.Ю. NBIC-конвергенция и ее влияние на развитие современной науки // Социально-политические науки. 2018. - №4. - C. 66-68.

\section{Referense}

1. Angelopoulou A., Mykoniatis K., Boyapati N. R. Industry 4.0: The use of simulation for human reliability assessment // Procedia Manu- facturing. - 2020. - №42. - P. 296-301. DOI:10.1016/j.promfg.2020.02.094.

2. Brocal F., González C., Komljenovic D., Katina P.F., Sebastián M.A. Emerging Risk Management in Industry 4.0: An Approach to Improve Organizational and Human Performance in the Complex Systems // Complexity. - 2019. №1-13. - DOI:10.1155/2019/2089763.

3. Kolbachev E., Kolbacheva T. Human Factor and Working Out of NBIC-Technologies // AHFE 2018: Advances in Manufacturing, Production Management and Process Control. - 2019. - Vol. 793. - P. 179-190. DOI:10.1007/978-3-319-94196-7_17.

4. Maksimović M., Vujović V., Perišić B. Do It Yourself solution of Internet of Things Healthcare System: Measuring body parameters and environmental parameters affecting health // Journal of Information Systems Engineering \& Management. — 2016. — №1 (1). — P. 25-39. DOI:10.20897/lectito.201607.

5. Mikulášková J., Čambál M., Polakovič L., Urbanovičová P. Spiral Management: New Concept of the Social Systems Management// Networked Business Models in the Circular Economy. - 2020. - P. 174-199. DOI:10.2507/29th.daaam.proceedings.131.

6. Nocker M., Sena V. Big Data and Human Resources Management: The Rise of Talent Analytics // Social Sciences. - 2019. — №8 (10). P. 273. - DOI:10.3390/socsci8100273.

7. Reunanen T. Human Factor in Time Management // Procedia Manufacturing. - 2015. №3. - P. 709-716. - DOI:10.1016/j.promfg.2015.07.311.

8. Robert M., Giuliani Ph., Gurau C. Implementing industry 4.0 real-time performance management systems: the case of Schneider Electric// Production Planning \& Control. - 2020. - DOI: 10.1080/09537287.2020.1810761.

9. Royakkers L., Timmer J., Kool L., Van Est R. Societal and ethical issues of digitization // Ethics and Information Technology. 2018. — №20. - P. 127-142. — DOI:10.1007/ s10676-018-9452-x.

10. Sassi I., Anter S., BekkhouchaA. An Overview of Big Data and Machine Learning Paradigms // Advances in Intelligent Systems and Computing. - 2019. - №915. - P. 237251. - DOI:10.1007/978-3-030-11928-7 21.

11. Som C., Hilty L.M. Qualitative risk assessment for converging technologies: nano-bio- 
info-cogno technologies // Proceedings of International Symposium on EcoTopia Science. 2007. - P. 1178-1181.

12. Tapus N., Manolache M.A. Integrated Decision Making using the Blockchain // Procedia Computer Science. - 2019. - №162. P. 587-595. - DOI:10.1016/j.procs.2019.12.027.

13. Klejner $G$. Sistemnaja paradigma i teorija predprijatija [System paradigm and the theory of the enterprise] // Voprosy jekonomiki [Economic issues]. - 2002. — №10. - Pp. 24-33.

14. Koval'chuk M. V., Narajkin O.S. Prirodopodobnye tehnologii - novye vozmozhnosti i novye ugrozy [Nature-like technologies - new opportunities and new threats] // Indeks bezopasnosti [Security index]. — 2016. — №22 (3-4). Pp. 118-119.

15. Koval'chuk M. V. Konvergencija nauk i tehnologij — proryv v budushhee // Rossijskie nanotehnologii [Convergence of sciences and technologies - a breakthrough in the future]. 2011. - №6 (13-23). — Pp. 455-465. DOI:10.31857/S0869-5873895455-465.

16. Koval'chuk M. V., Narajkin O.S., Jacishina E.B. Prirodopodobnye tehnologii: novye vozmozhnosti i novye vyzovy [Nature-like technologies: new opportunities and new challenges] // Vestnik Rossijskoj akademii nauk [Bulletin of the Russian Academy of Sciences]. - 2019. №89(5). - Pp. 455-465. - DOI:10.31857/ S0869-5873895455-465.

17. Kolbachev E.B. Proizvodstvennye sistemy mashinostroitel'nyh predprijatij i ih organizacionno-jekonomicheskaja jevoljucija [Production systems of machine-building enterprises and their organizational and economic evolution] // Problemy mashinostroenija i avtomatizacii [Problems of mechanical engineering and automation]. - 2016. - №2. Pp. 12-16.

18. Kornai Ja. Sistemnaja paradigma [System paradigm] // Voprosy jekonomiki [Economic issues]. - 2002. - №4. - Pp. 18-26.

19. Rudenskij O.V., Rybak O.P. Innovacionnaja civilizacija XXI veka: konvergencija i sinergija NBIC-tehnologij. Tendencii i prognozy 2015-2030 [Innovative civilization of the XXI century: convergence and synergy of NBIC technologies. Trends and forecasts 2015-2030] [Jelektronnyj resurs] // Informacionno-analiticheskij bjulleten' №3 [Information and analytical Bulletin №3]. — URL: http://www.vixri.ru/ wp-content/uploads/2011/08/inf3_2010.pdf.

20. Medvedev D.A., Prajd D. Fenomen NBIC-konvergencii: Real'nost' i ozhidanija [The phenomenon of NBIC convergence: Reality and expectations] // Filosofskie nauki [Philosophical sciences]. - 2008. — №1. Pp. 97-117.

21. Pavel'eva T. Ju. NBIC-konvergencija $\mathrm{i}$ ee vlijanie na razvitie sovremennoj nauki [NBIC-convergence and its influence on the development of modern science] // Social'no-politicheskie nauki [Socio-political Sciences]. 2018. — №4. — Pp. 66-68. 


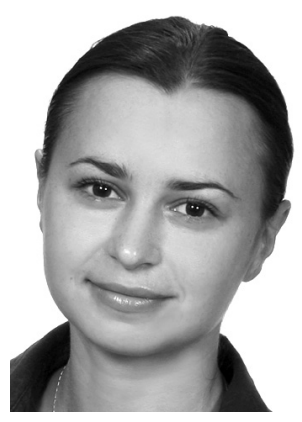

Пахомова Антонина Александровна - доктор экономических наук, профессор, доцент кафедры «Производственный и инновационный менеджмент» Южно-Российского государственного политехнического университета (НПИ).

Pakhomova Antonina Aleksandrovna - Doctor of Economic Sciences, Professor, Associate Professor of the Department «Production and Innovation Management», Platov South Russian State Polytechnic University (NPI).

341493, Ростовская обл., п. Персиановкий, ул. Садовая, 78

78 Sadovaya st., 341493, Persianovskiy, Rostov reg., Russia

E-mail: tivano@yandex.ru

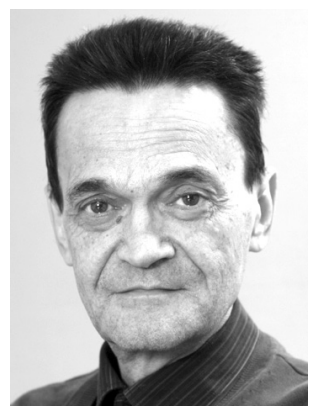

Федорчук Владимир Евгеньевич - доцент кафедры «Производственный и инновационный менеджмент» ЮжноРоссийского государственного политехнического университета (НПИ).

Fedorchuk Vladimir Evguenievich - Associate Professor of the Department «Production and Innovation Management», Platov South Russian State Polytechnic University (NPI).

346428 , г. Новочеркасск, ул. Просвещения, 132

132 Prosveshcheniya st., 346428, Novocherkassk, Russia E-mail: fiop_urgtu@mail.ru

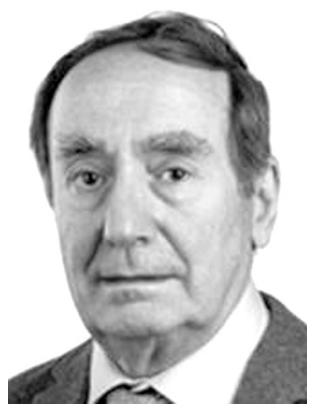

Кадар Иштван - кандидат технических наук, титульный профессор кафедры электроэнергетики Будапештского университета технологий и экономики.

Kadar Ishtvan - Candidate of Engineering Sciences, Titular Professor of the Department of Electric Power Engineering, Budapest University of Technology and Economics.

1111, г. Будапешт, Венгрия, Müegyetem rakpart 1-6

Müegyetem rakpart 1-6, 1111, Budapest, Hungary

E-mail:kadar.istvan@vet.bme.hu 